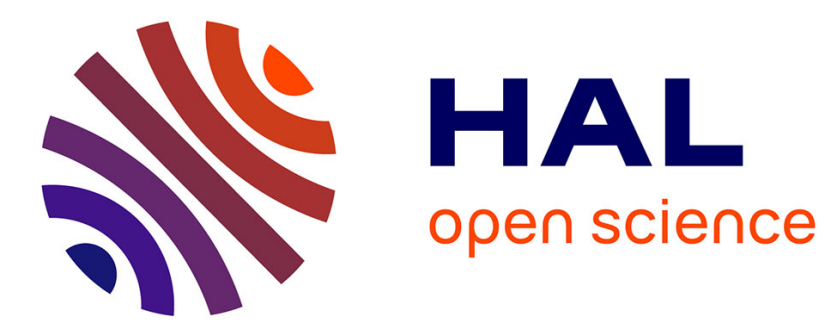

\title{
Image storage in coumarin-based copolymer thin films by photoinduced dimerization
}

Denis Gindre, Konstantinos Iliopoulos, Oksana Krupka, Emilie Champigny, Yohann Morille, Marc Sallé

\section{> To cite this version:}

Denis Gindre, Konstantinos Iliopoulos, Oksana Krupka, Emilie Champigny, Yohann Morille, et al.. Image storage in coumarin-based copolymer thin films by photoinduced dimerization. Optics Letters, 2013, 38 (22), pp.4636-4639. 10.1364/ol.38.004636 . hal-03344733

HAL Id: hal-03344733

https://univ-angers.hal.science/hal-03344733

Submitted on 15 Sep 2021

HAL is a multi-disciplinary open access archive for the deposit and dissemination of scientific research documents, whether they are published or not. The documents may come from teaching and research institutions in France or abroad, or from public or private research centers.
L'archive ouverte pluridisciplinaire HAL, est destinée au dépôt et à la diffusion de documents scientifiques de niveau recherche, publiés ou non, émanant des établissements d'enseignement et de recherche français ou étrangers, des laboratoires publics ou privés. 


\title{
Image storage in coumarin-based copolymer thin films by photoinduced dimerization
}

\author{
Denis Gindre, ${ }^{1, *}$ Konstantinos Iliopoulos,,${ }^{1,2}$ Oksana Krupka, ${ }^{1,3}$ Emilie Champigny, ${ }^{1}$ \\ Yohann Morille, ${ }^{1}$ and Marc Sallé ${ }^{1}$ \\ ${ }^{1}$ Université d'Angers, Laboratoire MOLTECH-Anjou, CNRS UMR 6200, 2 bd Lavoisier, 49045 Angers Cedex, France \\ ${ }^{2}$ Institute of Chemical Engineering Sciences, Foundation for Research and Technology Hellas (FORTH/ICE-HT), 26504 Patras, Greece \\ ${ }^{3}$ Department of Chemistry, Taras Shevchenko Kiev National University, Kiev, Ukraine \\ ${ }^{*}$ Corresponding author: denis.gindre@univ-angers.fr
}

Received July 16, 2013; revised September 19, 2013; accepted October 3, 2013; posted October 3, 2013 (Doc. ID 193981); published November 7, 2013

\begin{abstract}
We report a technique to encode grayscale digital images in thin films composed of copolymers containing coumarins. A nonlinear microscopy setup was implemented and two nonlinear optical processes were used to store and read information. A third-order process (two-photon absorption) was used to photoinduce a controlled dimerto-monomer ratio within a defined tiny volume in the material, which corresponds to each recorded bit of data. Moreover, a second-order process (second-harmonic generation) was used to read the stored information, which has been found to be highly dependent upon the monomer-to-dimer ratio. (c) 2013 Optical Society of America OCIS codes: (210.4770) Optical recording; (210.4810) Optical storage-recording materials; (210.0210) Optical data storage; (190.4400) Nonlinear optics, materials; (190.4710) Optical nonlinearities in organic materials; (180.4315) Nonlinear microscopy.

http://dx.doi.org/10.1364/OL.38.004636
\end{abstract}

The best technological solution for optical data storage (ODS) currently is the Blu-ray disk. This technology, based on the limitation of diffraction through decreasing the wavelength of the writing/reading laser beams, has increased storage capacity to about 25 GB in a single layer. Using double-layer disks the capacity of Blu-ray disks has been doubled, but the need for enhanced storing capacity/density and longer term storage solutions remains critical. Indeed, the volume of information that needs to be stored increases continuously. For instance, the ultra-high-definition (Ultra HD) 4K video technology, consisting of $7680 \times 4320$ pixels images, requires more than $100 \mathrm{~GB}$ for a $120 \mathrm{~min}$ movie. Progresses in laser diode technology $[1,2]$ could potentially provide capacities of $100 \mathrm{~GB}$ in a single layer using an ultraviolet solid-state laser of $385 \mathrm{~nm}$ wavelength, but cannot afford to meet the always increasing demand for storage and archiving. Up to now, processes used for writing information have been based on inducing phase or structural changes in the material by one-photon absorption. However, the density can be enhanced by new approaches, involving volumetric or 3D ODS. Holographic data storage is a potential technical solution [3-5], but has failed to penetrate the market despite several attempts in the last decade. Another approach, which has been employed recently to obtain microholographic recordings, has been based on utilizing a polymeric mixture [6].

Another promising approach to induce local changes in bulk materials is to use nonlinear (as for instance two-photon or multiphoton) processes. For example, two-photon absorption (TPA) occurs when the material absorbs two photons simultaneously. This nonlinear process requires high peak intensities (which are provided by focusing short laser pulses inside the volume of the material) and consequently occurs in a tiny spot of the focused beam where the intensity is higher. In order to achieve ODS through nonlinear processes, the nonlinear interaction should be followed by a modification at the molecular level. For example, this has been achieved recently by using fluorescent photochromic materials [7-9]. In a previous study [10], we demonstrated that disorder induced by TPA in oriented azobenzenes linked with polymers by means of trans-cis photoisomerization is a very promising method to achieve massive ODS. This approach is reversible [11] and offers the possibility to store grayscale images [12]. Unfortunately, these materials are not transparent over a broad spectrum in the visible region and multiple readout processes of the same stored data can gradually erase the information. Other approaches have also been developed to enhance storing capacities. For example, the number of degrees of freedom has been increased by employing gold nanorods, and more specifically, their longitudinal surface plasmon resonance. In this case, a five-dimensional patterning is obtained with wavelength and polarization multiplexing [ $\underline{13}]$. More recently, Church et al. reported the possibility to achieve a huge density of data by storage of the contents of an illustrated book by DNA encoding taking nucleotides $\mathrm{A}$ and $\mathrm{C}$ for the bit state 0 and nucleotides $\mathrm{G}$ and $\mathrm{T}$ for the bit state 1 [14].

An interesting concept of a molecular switch has been shown, which was based on photochemical reactions between diarylethylene and tetraarylcyclobutane by introducing fluorescent groups into the system [15]. Alternatively, a first demonstration of binary ODS in thin coumarin-substituted polymer films, supported by the photoinduced cyclodimerization of coumarin moieties, has been presented recently by our group [16], and the second-harmonic generation (SHG) efficiency of several coumarin-based polymers before and after photoinduced dimerization has been reported [17].

In this Letter, we extend our previous findings and demonstrate multilevel ODS via two-photon-induced photo-cross-linking of coumarin-based films. The storage medium consists of a copolymer synthesized from a coumarin-based methacrylate monomer and methyl 
methacrylate, with a copolymerization ratio of $n / m=$ $1 / 3$. For the needs of the recording, thin films (thickness $1.1 \mu \mathrm{m}$ ) were spin-coated on microscope glass slides and dried in vacuum for $5 \mathrm{~h}$ to eliminate any remaining solvent. In order to generate a SHG signal, the corona poling technique has been employed. The thin film is placed in a high electric field (about $500 \mathrm{kV} / \mathrm{m}$ ) while the plate is heated to $93^{\circ} \mathrm{C}$, corresponding to the glass transition temperature of this compound. During the cooling process to room temperature, the electric field is still applied in order to avoid the relaxation of the chromophores. In this way, the latter stay blocked in a direction perpendicular to the plane of the microscope slide.

The principle of the optical storage is presented in Fig. 1. The initial copolymer schematized by an orange ellipse [Fig. 1(b)] presents a high SHG response due to alignment of the chromophores by corona poling. When light of wavelength $\lambda$ between 300 and $350 \mathrm{~nm}$ illuminates the sample, cross-linking of the coumarins moieties is observed. This process is followed by a decrease of the characteristic absorption band of coumarin, which is attributed to the formation of a cyclobutane dimer from the coumarin double bond [Fig. 1(a)] as we have reported previously [17]. The dimer form, schematized by a blue ellipse in Fig. 1 , is less conjugated than the initial coumarin monomer, leading consequently to a local decrease of the SHG response. The SHG efficiency emanating from the latter areas was very low and has been attributed to the lowest SHG value (highest contrast) during the ODS. On the contrary, the SHG value corresponding to the nonirradiated areas was the highest SHG value during the recordings (zero contrast). Importantly, by properly adjusting the laser power and the exposure time, we have been able to control the dimerto-monomer ratio of chromophores in the irradiated areas (Fig. 1, schemes 3 and $3^{\prime}$ ). This approach gives the opportunity to modulate the SHG response of the material between the highest and the lowest SHG values

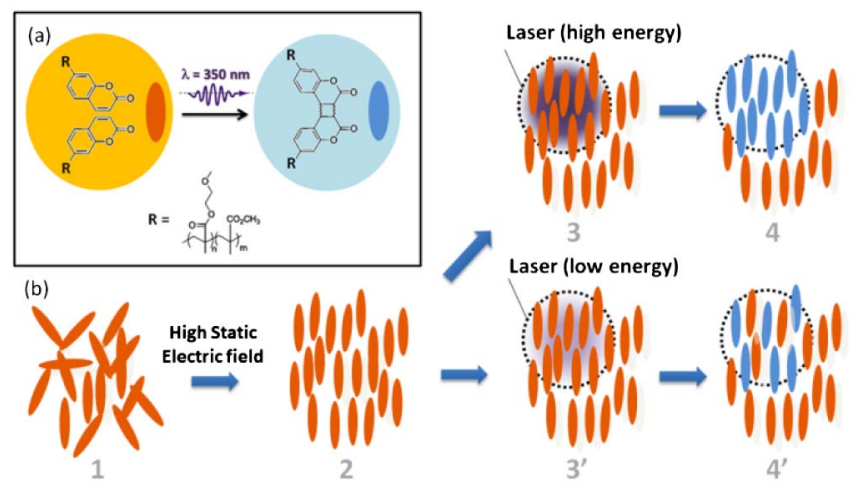

Fig. 1. (a) Molecular structure of the initial coumarin/ copolymer (schematized by the orange ellipse in the yellow circle) and the photo-cross-linked state of the coumarin/ copolymer (blue ellipse in the soft blue circle) obtained by ultraviolet irradiation. (b) Principle of analogic level storage. The oriented state of the copolymer (2) was induced from the initial disordered state (1) by using the corona poling technique. The laser is then focalized on a small area of the film. At a high power of the laser or long-time exposure (3), all molecules in the voxel become cross-linked (4). At a lower power or a lower exposure time $\left(3^{\prime}\right)$, only a part of the molecules becomes dimerized $\left(4^{\prime}\right)$. and as a result, the storing of grayscale images. During this procedure, we first determined the gray level of each pixel of the image to be stored and then calibrated the setup by attributing a specific exposure time or laser power to each gray level. It is worth noting that a clear advantage over the previously reported optically induced local isomerization process of azobenzenes [10-12] lies in the fact that coumarin photodimerization allows reading the information in a transparent region of the spectrum, and therefore, does not result in any modification of the stored data during the reading phase. Finally, it should be noted that no degradation/modification of the thin films has been observed by atomic force microscope (AFM) measurements in the irradiated areas and under the laser powers and exposure times used.

A schematic diagram of the nonlinear microscopy optical setup is presented in Fig. 2(a). The laser source is a Ti:sapphire laser (Tsunami, Spectra Physics) pumped by a $10 \mathrm{~W}$ solid-state laser (Millenia Xs, Spectra Physics). Wavelength is fixed at $700 \mathrm{~nm}$ during the writing process (TPA at $350 \mathrm{~nm}$ ) and at $800 \mathrm{~nm}$ for the readout process, which corresponds to a transparent spectral region of the copolymer for both the fundamental wavelength and the TPA process. The pulse duration is about $120 \mathrm{fs}$ and the repetition pulse rate is $80 \mathrm{MHz}$. A broadband, half-wave plate (Edmund Optics, XV745) mounted on a motorized rotation stage and a Glan-Taylor polarizer (P) were used to control the intensity of the incident infrared light beam. A telescope (TS) increased the diameter of the beam to underfill the microscope objective (x20, NA 0.25, Olympus), in order to improve the axial resolution. A short-pass filter (BG39, Schott) has been placed after the sample to block the residual incident laser beam. The sample was placed on XYZ motorized stages (Newport) controlled by an eight-axis Motion Controller/Driver (XPS, Newport). The voxel length (typically $3 \mu \mathrm{m}$ with this microscope objective) is larger than the film thickness, but the efficiencies of the photoinduced cross-linking of coumarins and the SHG signal are second-order nonlinear effects and are dramatically dependent on the focusing. During recording, this step is very important to always stay focused on the thin film layer of coumarin. However, it is not always obvious that the plane defined by the two axes $x$ and $y$ [see Fig. 2(b)] of the system of coordinates $x y z$ is coplanar with the plane of the film. To circumvent this drawback, we address a specific feature of the controller to allow
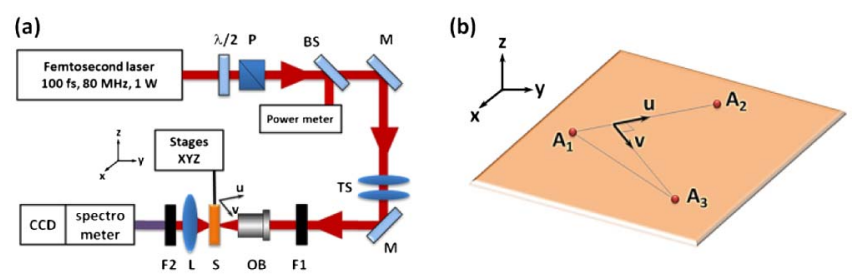

Fig. 2. (a) Optical setup. P, polarizer; BS, beam splitter; M, mirrors; TS, telescope; F1, long-pass filter; OB, microscope objective; S, sample; L, collection lens; F2, short-pass filter; CCD, charge-coupled device camera. (b) Definition of the plane of the sample. The orthonormal coordinate system $x y z$ is defined by the translation stages, and $A_{1}, A_{2}$, and $A_{3}$ are three points on the thin film, which permit defining the plane equation $(u, v)$ of the sample. 
simultaneous movement of the three stages. More specifically, we first defined three arbitrary nonaligned points into the sample: $A_{1}, A_{2}$, and $A_{3}$ [Fig. 2(b)]. From their coordinates, the unit vectors of the plane of the sample $\vec{u}$ and $\vec{v}$ were defined by means of the following equation:

$$
\vec{u}=\frac{\overrightarrow{A_{1} A_{2}}}{\left\|\overrightarrow{A_{1} A_{2}}\right\|} ; \vec{v}=\frac{\overrightarrow{A_{1} A_{3}}-\vec{u}\left(\overrightarrow{A_{1} A_{3}} \cdot \vec{u}\right)}{\left\|\overrightarrow{A_{1} A_{3}}-\vec{u}\left(\overrightarrow{A_{1} A_{3}} \cdot \vec{u}\right)\right\|}
$$

Then, with the plane of the sample known, the program calculates a transformation matrix, which transforms every movement on the plane of the sample to synchronous movement of the stages XYZ, thus allowing perfect focus of the laser beam on the sample throughout the recording. During reading of the data, the detection of the SHG signal is carried out with a fast-speed, low-noise, cooled CCD camera (Princeton) attached at the exit of a spectrometer (Acton) with its grating tuned to detect the SHG signal. The recorded spectral window depth is about $60 \mathrm{~nm}$ and calculation of the SHG level is carried out by numerically integrating all the parts of the spectrum corresponding to the SHG signal. A Visual $\mathrm{C}++$ program has been written to control the three grouped translation stages, the spectrometer, and the CCD camera.

As mentioned previously, in order to write an image into the material, a calibration is needed to be done. In this case, we performed this step by measuring the cross-linking of the coumarins as a function of the energy given to the material in the voxel volume. This procedure has been done by writing a circular gradient [see Fig. 3(a), upper part] containing 256 gray levels. The power of the incident beam has been fixed at $23 \mathrm{~mW}$ $(\lambda=700 \mathrm{~nm})$ and for each pixel the exposure time has been modified between 0 (no irradiation resulting in no modifications) and $1020 \mathrm{~ms}(255 \times 4)$. Another possibility would have been to modify the laser intensity for each pixel instead of the exposure time. However, this approach would have the drawback that the mechanical control of the half-wavelength would be slower compared with the control of the shutter, so a very long time would be required to write, for example, a $100 \times 100$ pixel image. (a)

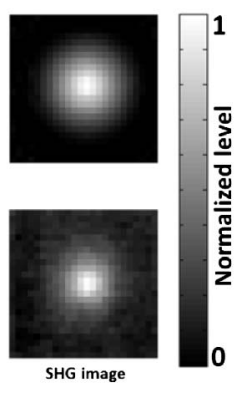

(b)

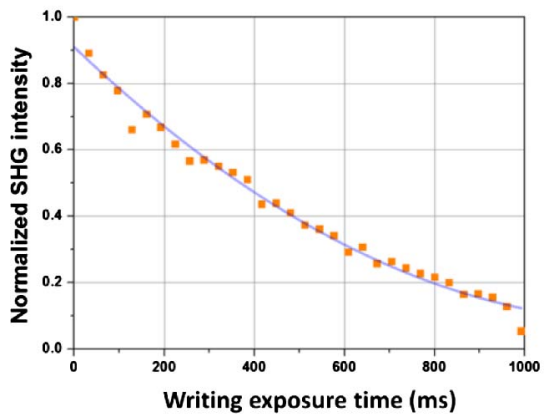

Fig. 3. (a) Reference image (upper part) and SHG image (lower part) used for calibration. (b) Normalized SHG intensity as a function of the exposure time during the writing process.
The distance between two adjacent spots in the sample has been fixed at $2 \mu \mathrm{m}$. Then, in order to scan the SHG efficiency in the area of the circular gradient, the wavelength of the laser has been changed to $800 \mathrm{~nm}$ and the laser power has been fixed at $5 \mathrm{~mW}$. Thus, away from the two-photon resonance and with a much lower power than that needed to record, we were sure that no other modifications would take place during the scan. The scanning was carried out by reading the SHG horizontally along the image shown in the lower part of Fig. 3(a). The distance between adjacent lines was chosen to be $2 \mu \mathrm{m}$, which is sufficient resolution for the calibration purposes. In the very beginning of the scanning of each line, the translation stages undergo an accelerating phase, while they continue with constant velocity when the acceleration phase is completed. This behavior can have a destructive effect on the images obtained because the distance between two adjacent pixels would not be the same for all the positions in the SHG image. In order to solve this problem, we started the stages' motion outside the area shown in the SHG image in Fig. 3(a). A synchronization signal was emitted when a translation stage was passing from a specific position corresponding to the border of the area. At this time, the acceleration phase was finished and the velocity of the stages was constant. Then, while the stage was passing through the desired area, a large number of spectra were recorded. After numerical integration of each of them and within the SHG bandwidth, we obtained the SHG image shown in the lower part of Fig. 3(a). It is obvious that in this case, the dimension in pixels of the image is related to the integration time of the spectra and the velocity of the stages throughout the scanning procedure. By comparison between the two images in Fig. 3(a), the relation between the exposure time and the SHG decrease has been established and is shown in Fig. 3(b). It can be seen that for the zero-exposure time [corresponding to the center of the images in Fig. 3(a)] the maximum SHG value has been obtained as expected, whereas a fast decrease is observed as the exposure time increases. The lowest SHG value has been obtained for the longest times of exposure corresponding to the borders of the images in Fig. 3(a), as predicted. However, we could not achieve a zero SHG signal. It was also practically impossible to obtain $100 \%$ transformation of coumarin dimers to monomers; and even if complete transformation could have been achieved, some SHG efficiency would have remained, corresponding to the very low SHG efficiency of the dimers. The line passing though the points in Fig. 3(b) is a third-order polynomial fit that matches the behavior of our experimental data perfectly.

In order to show the potential of this approach, we have chosen to encode a grayscale image $(207 \times 102$ pixels) containing the Lena image and the logo of University of Angers [Fig. 4(b)]. Prior to the recording, a scan of SHG efficiency was performed in the area where the recording was intended to take place (area size: $210 \mu \mathrm{m} \times$ $100 \mu \mathrm{m})$. During this experiment, the laser beam was adjusted at $800 \mathrm{~nm}, 5 \mathrm{~mW}$, as in the previous experiment and a rapid scan of 150 horizontal lines at a velocity of $10 \mu \mathrm{m} / \mathrm{s}$ was applied. At this speed and with an acquisition time of $100 \mathrm{~ms}$ for each SHG spectrum, a $200 \times 150$ pixel SHG image was obtained [Fig. 4(a)]. It can be seen 
(a)
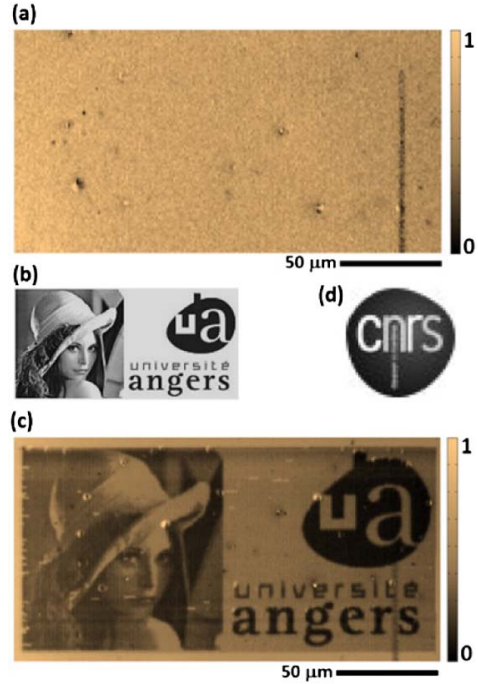

(e)

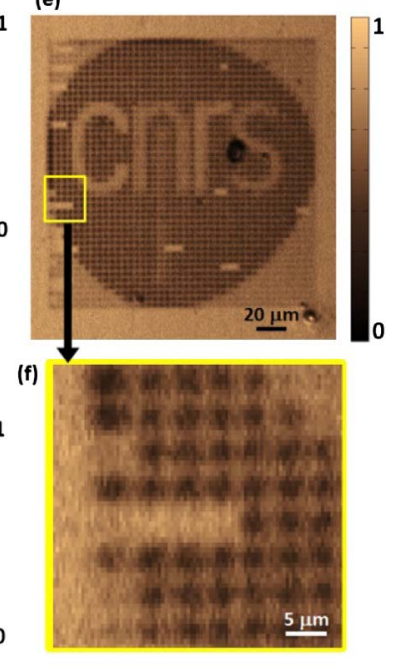

Fig. 4. (a) Normalized SHG image obtained after a scan of the initial area. (b) Reference image. (c) Normalized SHG image obtained by scanning the area after the recording. (d) Reference image (CNRS logo). (e) SHG image of the CNRS logo. (f) Zoom of an area in the SHG image.

that the SHG signal is nearly constant all over the surface, except in an area where a short line was written initially. The latter is done in order to make easier the identification of the area during our experiments, as the recorded data are completely invisible by means of conventional optical microscopy. During the second phase, we changed the wavelength of the laser to $695 \mathrm{~nm}$, the power was fixed at $26 \mathrm{~mW}$, and the exposure time for each pixel was tuned between 0 and $1020 \mathrm{~ms}$ according to the calibration procedure performed previously. The distance between each recorded bit was fixed at $1 \mu \mathrm{m}$, below the diffraction limit. The third phase consisted of re-scanning the same area $(800 \mathrm{~nm}, 5 \mathrm{~mW})$ with 330 lines and at a slower speed $(5 \mu \mathrm{m} / \mathrm{s})$ in order to increase the resolution of the SHG image $(350 \times 330$ pixels $)$. The final image containing the stored information is shown in Fig. 4(c) in which very good contrast can be seen between the stored data and the background. Next, a 43 pixel, squareshaped, digitalized, grayscale image [Fig. 4(d)] of the logo of the CRNS (Centre National de la Recherche Scientifique) was stored, for which the spacing between two pixels corresponded to $4 \mu \mathrm{m}$ on the thin film. The obtained SHG image can be seen in Fig. 4(e) and a zoom of the SHG image is provided to illustrate the resolution of the stored image [Fig. $\underline{4(\mathrm{f}) \text { ]. }}$.

In conclusion, we have presented in this study a new approach to encode data using multiple-level recording with coumarin-based copolymers. The principle is based on modifying the pixel-by-pixel dimerization ratio through TPA processes. This ratio has a direct impact on the SHG efficiency. Consequently, the data can be readily collected by scanning the SHG all over the area where the information is stored. To illustrate the efficiency of this process, two different examples of highcontrast storage imaging using coumarin films have been presented. Finally, it is worth noting that the stored data are undetectable by usual linear optical techniques. Studies are therefore in progress toward extension of these results to the field of hidden storage. Moreover, the possibility to increase the density of data by performing volume data storage in thicker coumarin films, as well as improving the reading/recording times, is currently under investigation by our group and it can possibly open new prospects for employing these materials in storage applications.

K. I. acknowledges the support from the European Commission and the General Secretariat for Research and Technology (Greece) for a National Strategic Reference Framework (NSRF) Project (PE3-(1612)). This work was supported by the Région des Pays de la Loire (Projects MolTechAnjou and Movamol).

\section{References}

1. S. Chu, G. Wang, W. Zhou, Y. Lin, L. Chernyak, J. Zhao, J. Kong, L. Li, J. Ren, and J. Liu, Nat. Nanotechnol. 6, 506 (2011).

2. Y. Yamashita, M. Kuwabara, K. Torii, and H. Yoshida, Opt. Express 21, 3133 (2013).

3. T. Watanabe and M. Watanabe, Opt. Express 19, 24147 (2011).

4. Y.-C. Jeong, B. Jung, D. Ahn, and J.-K. Park, Opt. Express 18, 25008 (2010).

5. J. F. Heanue, M. C. Bashaw, and L. Hesselink, Science 265, 749 (1994).

6. R. Castagna, F. Vita, D. E. Lucchetta, L. Criante, and F. Simoni, Adv. Mater. 21, 589 (2009).

7. C. C. Corredor, Z.-L. Huang, and K. D. Belfield, Adv. Mater. 18, 2910 (2006).

8. J. Lott, C. Ryan, B. Valle, J. R. Johnson, D. A. Schiraldi, J. Shan, K. D. Singer, and C. Weder, Adv. Mater. 23, 2425 (2011).

9. S. Kawata and Y. Kawata, Chem. Rev. 100, 1777 (2000).

10. D. Gindre, A. Boeglin, G. Taupier, O. Crégut, J.-P. Vola, A. Barsella, L. Mager, A. Fort, and K. D. Dorkenoo, J. Opt. Soc. Am. B 24, 532 (2007).

11. D. Gindre, A. Boeglin, A. Fort, L. Mager, and K. D. Dorkenoo, Opt. Express 14, 9896 (2006).

12. D. Gindre, I. Ka, A. Boeglin, A. Fort, and K. D. Dorkenoo, Appl. Phys. Lett. 90, 094103 (2007).

13. P. Zijlstra, J. W. M. Chon, and M. Gu, Nature 459, 410 (2009).

14. G. M. Church, Y. Gao, and S. Kosuri, Science 337, 1628 (2012).

15. F. Li, J. Zhuang, G. Jiang, H. Tang, A. Xia, L. Jiang, Y. Song, Y. Li, and D. Zhu, Chem. Mater. 20, 1194 (2008).

16. K. Iliopoulos, O. Krupka, D. Gindre, and M. Sallé, J. Am. Chem. Soc. 132, 14343 (2010).

17. Z. Essaïdi, O. Krupka, K. Iliopoulos, E. Champigny, B. Sahraoui, M. Sallé, and D. Gindre, Opt. Mater. 35, 576 (2013). 